\title{
Investigation of phase equations for anaerobic digestion process of anaerobic bioreactor landfill
}

\author{
Priyanka R. Shinde ${ }^{1}$, Sandip. T. Mali ${ }^{2 *}$ \\ ${ }^{1}$ Department of Technology, Savitribai Phule Pune University, Pune, 411007, India \\ ${ }^{2}$ Department of Civil Engineering, Pimpri Chinchwad College of Engineering, Nigdi, 411044, Pune, India
}

\begin{abstract}
The anaerobic digestion process consists of four phases hydrolysis, acidogenesis, acetogenesis and methanogenesis. In the present work anaerobic digestion process of waste from anaerobic bioreactor landfill is considered and leachate is analyzed chemically for 268 days and out of all 17 parameters, seven major chemical parameters are studied for two reactors R4 and R5 for different reactor conditions and their phase distribution is done by two methods standard and transitory method. These phase distribution also gives phase wise equation, for optimization of entire process each phase equation is essential. The accuracy of curve equations is defined by R2 value. These equations help in formation of mathematical model using MATLAB. This model is useful for defining phase values of parameters. Further quality and quantity of chemical parameters are also defined by these phase values.
\end{abstract}

Keywords: Anaerobic digestion, Hydrolysis, Acidogenesis, Acetogenesis, Methanogenesis, Anaerobic bioreactor landfill, leachate.

\section{Introduction}

Anaerobic digestion is the process occurs in the absence of oxygen by which organic waste is broken down to produce biogas. Anaerobic digestion is a series of processes produced by bacteria that transform organic materials into biogas through four different stages including hydrolysis, acidogenesis, acetogenesis and methanogenesis. ${ }^{[1]}$ Organic processes are broken down step by step through these four stages towards the development of methane. In the first step of hydrolysis complex macromolecules and components are found in organic matter is converted into simple sugars, long chain fatty acids and amino acids, and conversion of these soluble micro molecules into volatile acetic acids, fatty acids, $\mathrm{H}_{2}$ and $\mathrm{CO}_{2}$ is occurs in the second stage called as acidogenesis. The transformation of volatile fatty acids to more acetic acid, $\mathrm{CO}_{2}$ and $\mathrm{H}_{2} \mathrm{~S}$ gas is formed in third stage of acetogenesis. The final stage of methanogenesis has the potential to generate methane by using $\mathrm{CO}_{2}$ and $\mathrm{H}_{2} \mathrm{~S}$ gas; otherwise acetic acid generated either in the second or third phases. Therefore, the Anaerobic Digestion process, if poorly controlled, will become inefficient and result in a reduced output of biogas, thus there is more room for further development of the production rate of biogas.
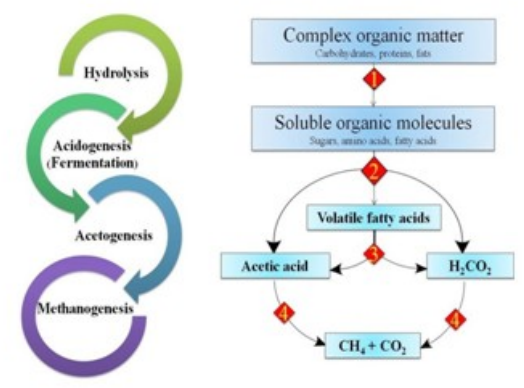

Figure 1:-Anaerobic digestion Process (Source: B.P. 2017)

In the anaerobic digestion process there are total four stages Hydrolysis, Acetogenesis, Acidogenesis and methanogenesis. The phase distribution of each parameter from leachate chemical analysis is done on the basis of vesilind's standard values for phase distribution also the another method is on the basis of transitory changes in values of parameter leads to change in phase of anaerobic digestion.

\footnotetext{
*Corresponding author: sandipmali05@gmail.com
} 


\section{Materials and methods}

\subsection{Reactor configuration}

Two groups of simulated Anaerobic Bioreactor Landfill were used in this study; first group for mixed MSWs with three Bioreactors (R1, R2, and R3) and the other group was consist of two Bioreactors (R4 and R5) of compostable MSWs. Various combinations of operating parameters have been aeration with introduction of aerobic microbial culture, anaerobic sludge, mixing of coarse gravel, soil cover as a intermediate layer and varying leachate circulation. For study purposes, we selected the reactor R5 ${ }^{[2]}$.

\subsection{Reactor conditions}

Bioreactor R4, R5- Consist of Re-circulation of leachate simulations and bioreactor had aeration with addition of aerobic microbial culture, gravel mixing and introduction of sludge in re-circulated leachate intermediately.

The bioreactor was constructed of acrylic plastic with a thickness of $5 \mathrm{~mm}$, an internal diameter of $0.3 \mathrm{~m}$ and a height of $1.2 \mathrm{~m}$ and was fitted with three ports. For leachate drainage and sampling there was first port was. For collecting gas samples and leachate recirculation other two ports are provided. A half-cut plastic ball was held at the center of the bottom above the leachate outlet to keep the leachate outlet from collapsing. The waste level in each reactor was $1 \mathrm{~m}$ and the freeboard was $0.1 \mathrm{~m} .50 \mathrm{~mm}$ thick layer of coarse gravel was put at the bottom of the reactor for leachate drainage. The waste was packed in six layers with a thickness of 0.15 $\mathrm{m}$ and $10 \mathrm{~mm}$ thick layers of waste after each layer. 50 $\mathrm{mm}$ of soil layer was supplied at the top of the waste. The porous pipe was placed in the center of the waste mass to capture biogas. Every reactor was opened for sampling and temperature measurement at a distance of $0.30 \mathrm{~m}$ from the bottom of $50 \mathrm{~mm}$ in diameter. To prevent algal production of the reactors were wrapped in black cloth.

The reactors were kept in a closed room and run under atmospheric conditions. The temperature variability over the length of the experiment (270 days) ranged from $22.9^{\circ} \mathrm{C}$ to $39.4^{\circ} \mathrm{C}$ with a mean temperature of $29.8^{\circ} \mathrm{C}$. Leachate was collected in a container (vol. 13 $\mathrm{L}$ ) at the bottom of the reactor and transferred to an overhead container (a submersible pump made by Futura Industries India, model - KR 1900). The leachate was pumped into the reactor through the upper layer of the soil using a PVC pipe which is perforated. In the same day of charging, the bioreactor R4 was coated with an acrylic film. Waste material worked under anaerobic conditions for 270 days. After 14 days of aerobic treatment, the bioreactors R3 and R5 were sealed.

\subsection{Analysis of Reactor}

Leachate was collected and stored in $1 \mathrm{~L}$ air tight plastic bottle. The bottles were stored at $-4^{\circ} \mathrm{C}$ in refrigerator. Chemical analysis of leachate was carried out after every 7 days for first 60 days (on every Monday analysis was started) and after that every 14 days up to 90 days and afterwards every 21 days to till the end of experimentation, as there was not too much variation in the characteristics of leachate. Leachate analysis was performed for 17 parameters $(\mathrm{pH}$, electrical conductivity(EC), total solids (TS), total dissolved solids (TDS), volatile solids (VS), solids (FS), biochemical oxygen demand for 3 days $\left(\mathrm{BOD}_{3}\right)$, chemical oxygen demand (COD), total kejeldhal nitrogen (TKN), chloride, oxidation reduction potential (ORP), sulphate, sodium (Na), calcium $(\mathrm{Ca})$, potassium $(\mathrm{K})$, volatile fatty acids (VFA) and alkalinity. All the analysis was carried as per standard methods.

\section{Results and discussion}

\subsection{Standard method of phase distribution}

Based on vesilind's table the standard ranges of parameters are distributed as follows:

Landfill constituent concentration ranges as a function of the degree of stabilization:

Table 1: Anaerobic digestion Process phase values for parameters

\begin{tabular}{|c|c|c|c|c|}
\hline Parameter & Transition & Acid formation & $\begin{array}{c}\text { Methane } \\
\text { fermentation }\end{array}$ & Maturation \\
\hline COD (mgL $\left.{ }^{-1}\right)$ & $480-18000$ & $1500-71000$ & $580-9760$ & $31-900$ \\
\hline $\begin{array}{c}\text { Total volatile } \\
\text { acids (mgL }\end{array}$ & $100-3000$ & $3000-18800$ & $250-4000$ & 0 \\
\hline $\begin{array}{c}\text { Ammonia }\left(\mathrm{mgL}^{-1}\right. \\
\text { ) }\end{array}$ & $120-125$ & $2-1030$ & $6-430$ & $6-430$ \\
\hline $\mathrm{pH}\left(\mathrm{mgL}^{-1}\right)$ & 6.7 & $4.7-7.7$ & $6.3-8.8$ & $7.1-8.8$ \\
\hline $\begin{array}{c}\text { Conductivity } \\
\left(\mathrm{mgL}^{-1}\right)\end{array}$ & $2450-3310$ & $1600-17100$ & $2900-7700$ & $1400-4500$ \\
\hline
\end{tabular}

(Source: Vesilind et al., 2003, pp. - 120)

\subsection{Transitory phase distribution}

In this method the phase distribution is done on the basis of transitory change in the parameter values and that forms the phases of anaerobic digestion.

After phase distribution of anaerobic digestion process comparison between both of these parameters is done as result of that we get the comparison of equation and $\mathrm{R}^{2}$ value for each phase that comparison 
will decide the best suitable method for phase distribution.

\section{Colour code for Phase Distribution:}

Blue- Phase I

Red- Phase II

Yellow- Phase III

Violet- Phase IV

Green- Phase V

Results for R4 reactor Transitory phase distribution:

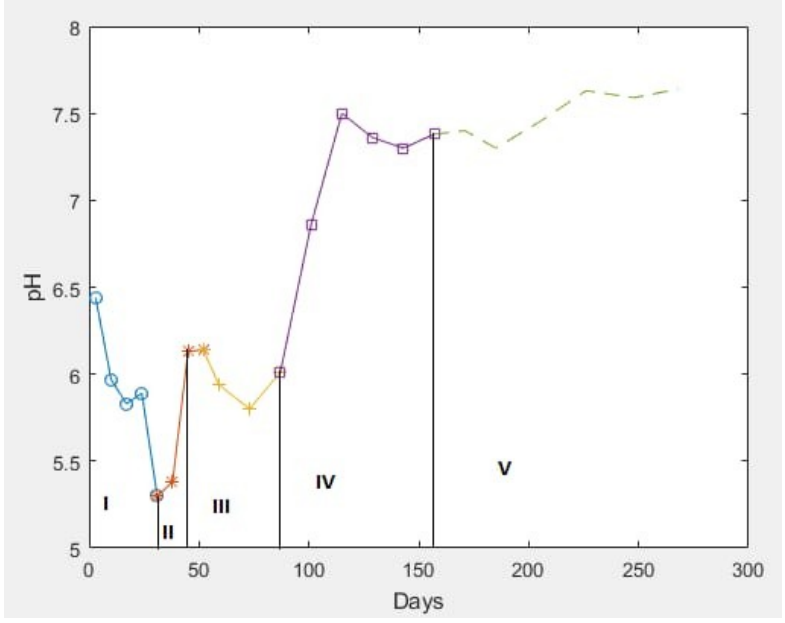

Figure 2:-pH Transitory phase distribution

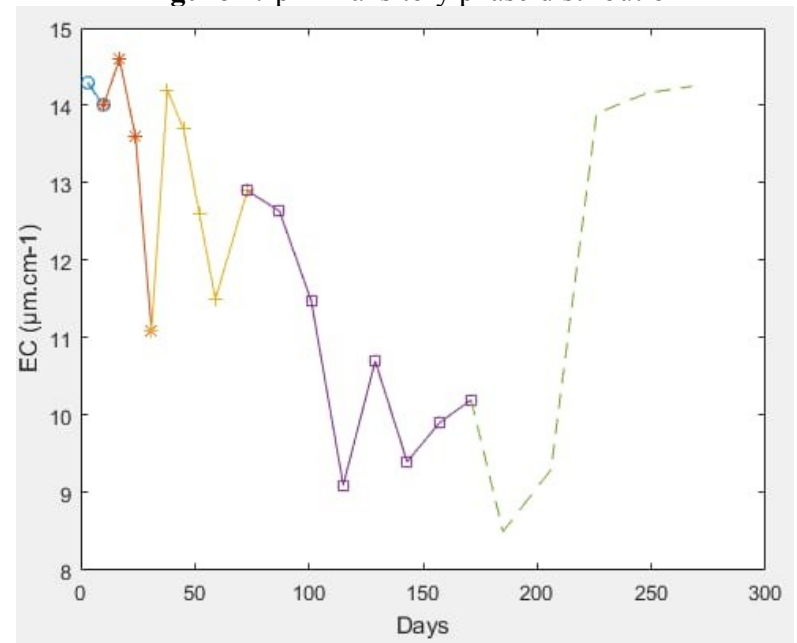

Figure 3:- EC Transitory phase distribution

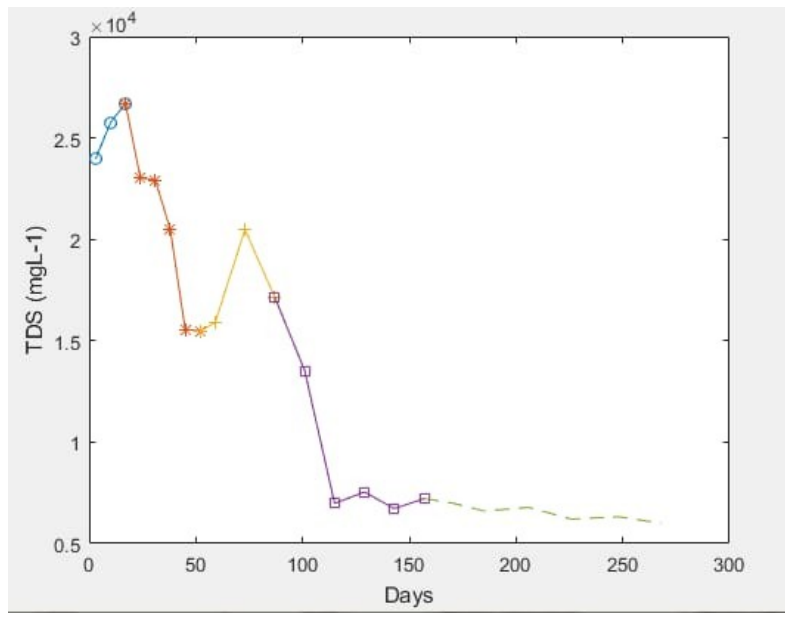

Figure 4:- TDS Transitory phase distribution

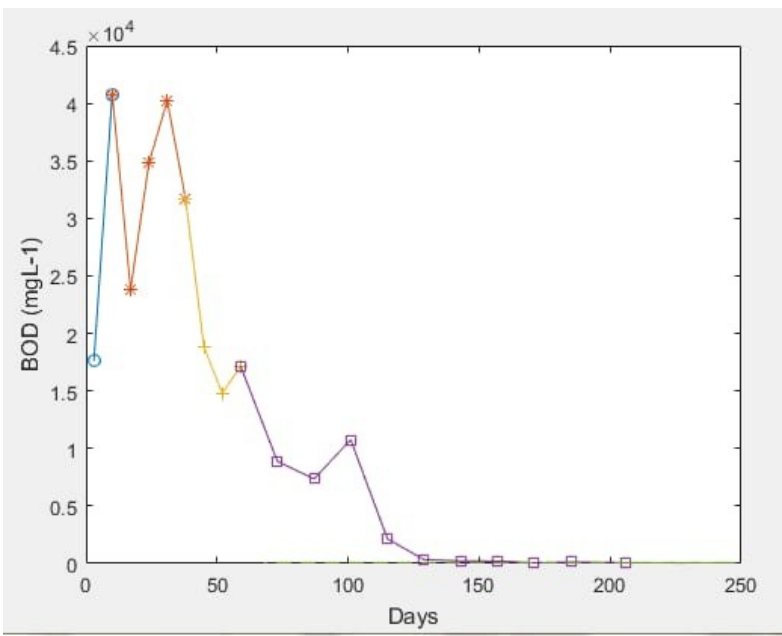

Figure 5:- BOD Transitory phase distribution

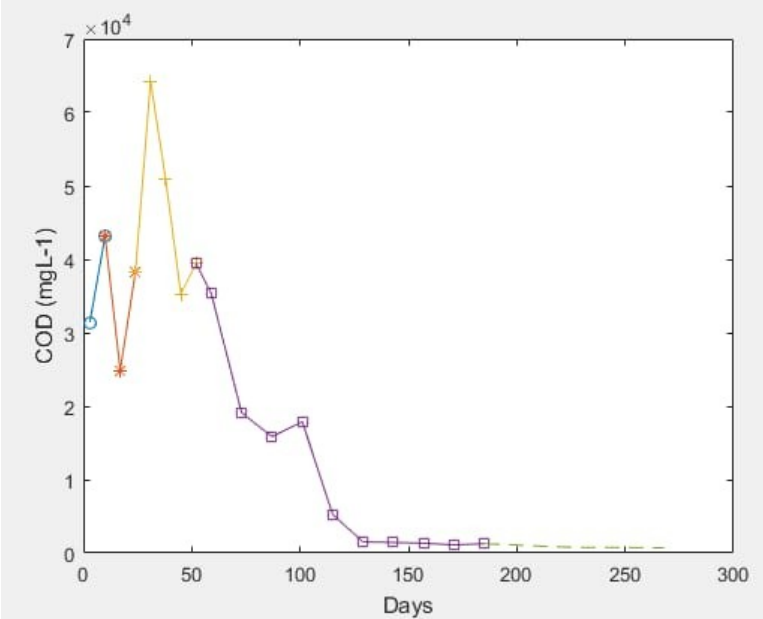

Figure 6:- COD Transitory phase distribution 


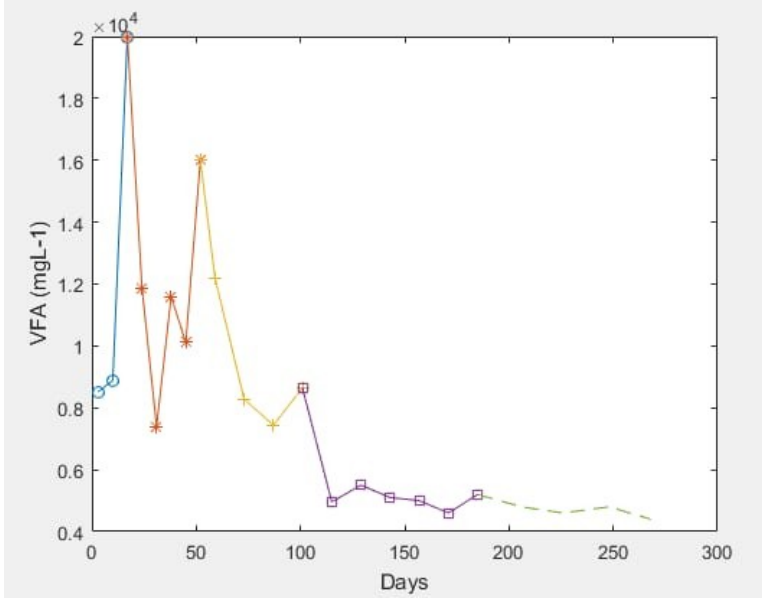

Figure 7:- VFA Transitory phase distribution

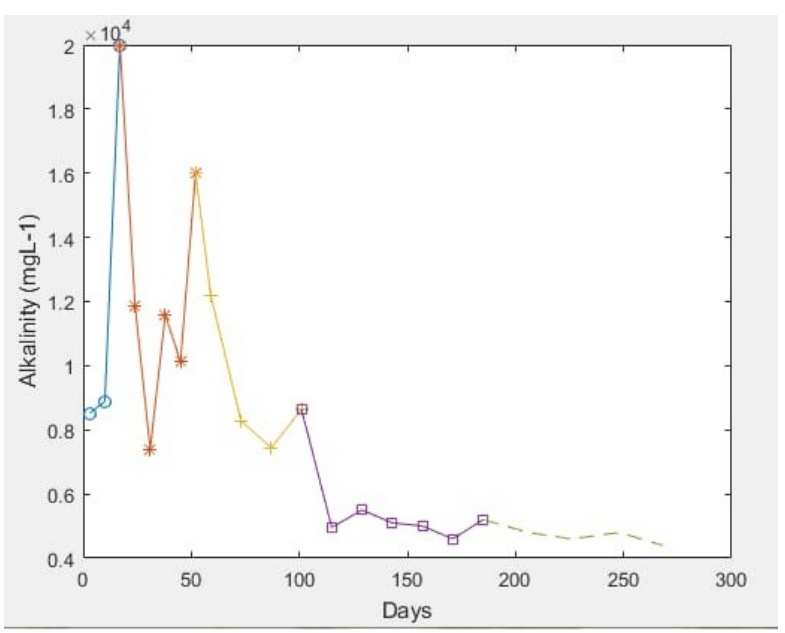

Figure 8:- Alkalinity Transitory phase distribution

Results for reactor R5 transitory phase distribution:

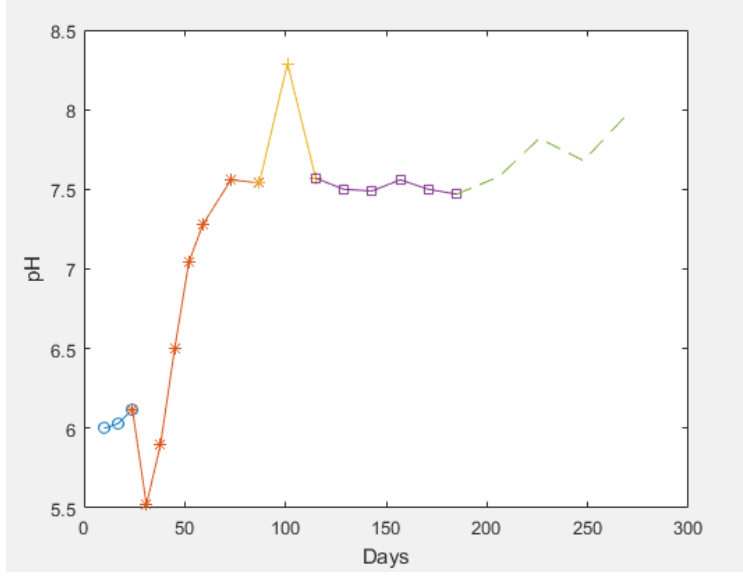

Figure 9:- $\mathrm{pH}$ transitory phase distribution

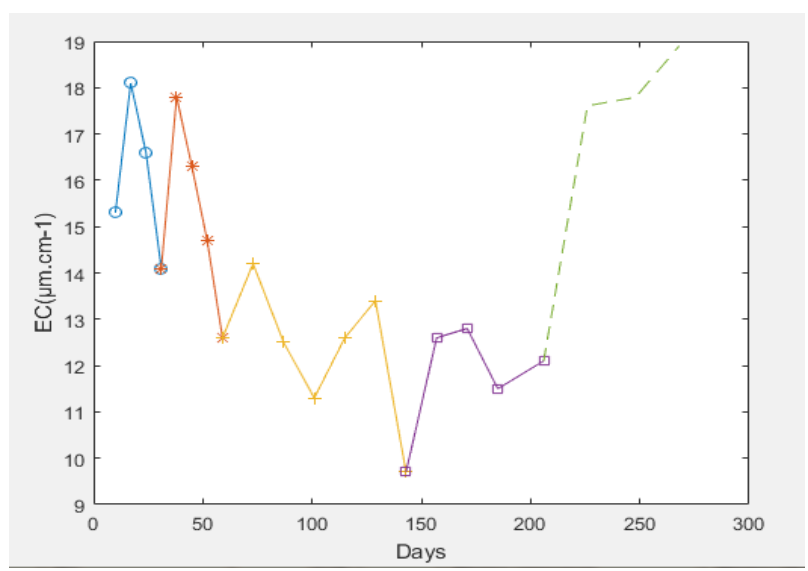

Figure 10:- EC transitory phase distribution

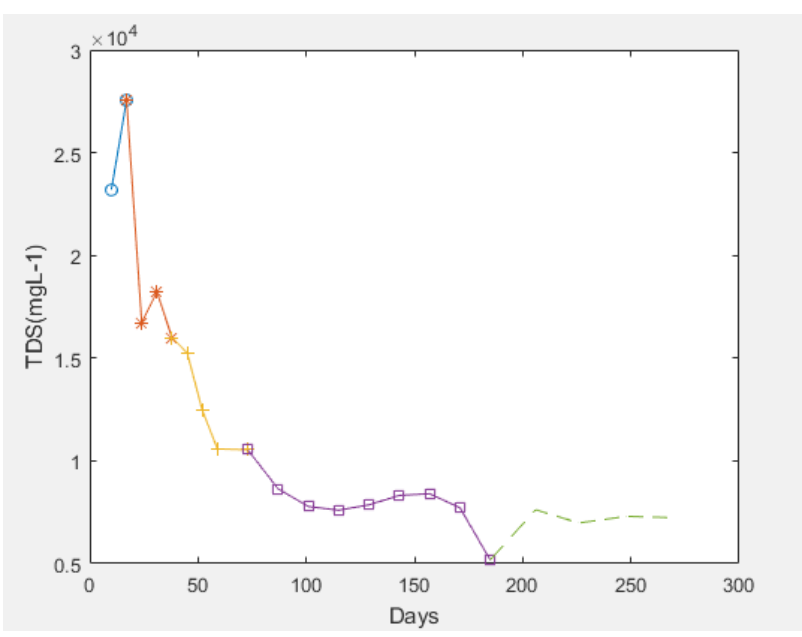

Figure 11:- TDS transitory phase distribution

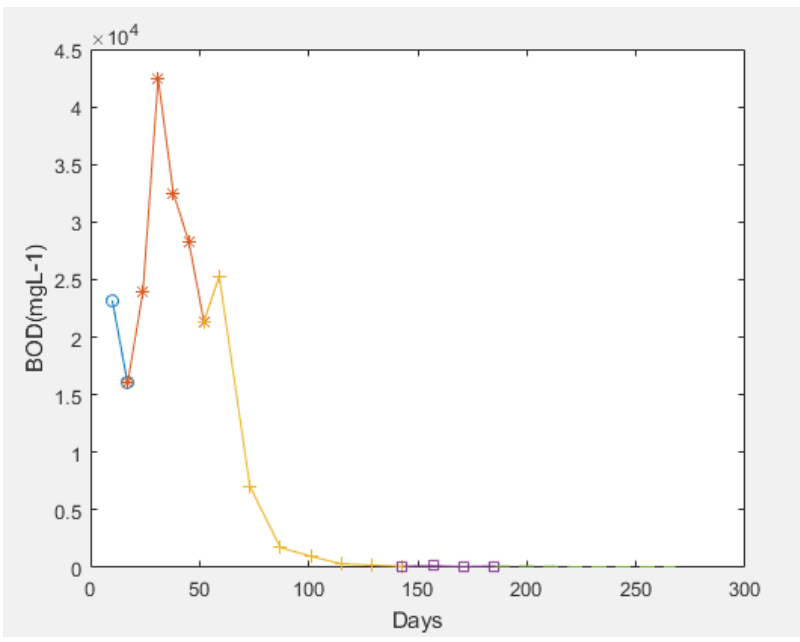

Figure 12:- BOD transitory phase distribution 




Figure 13:- COD transitory phase distribution

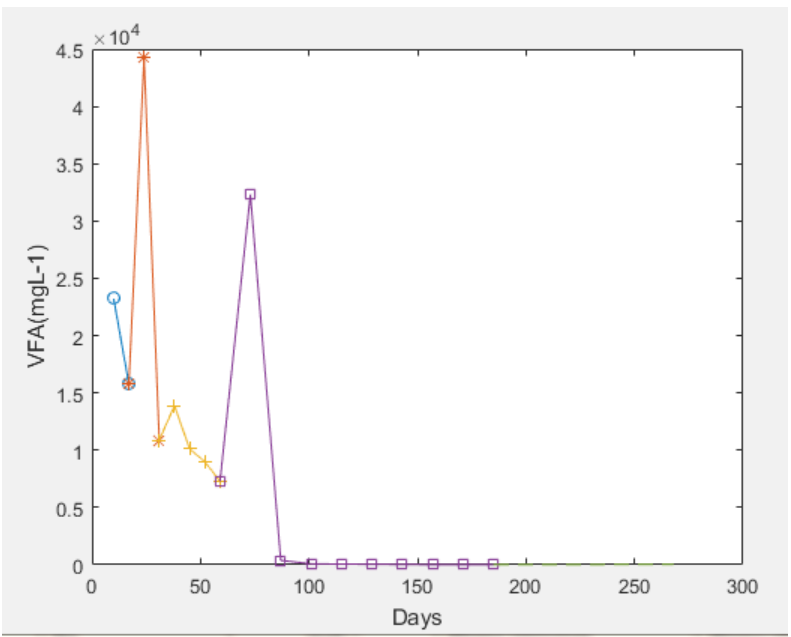

Figure 14:- VFA transitory phase distribution

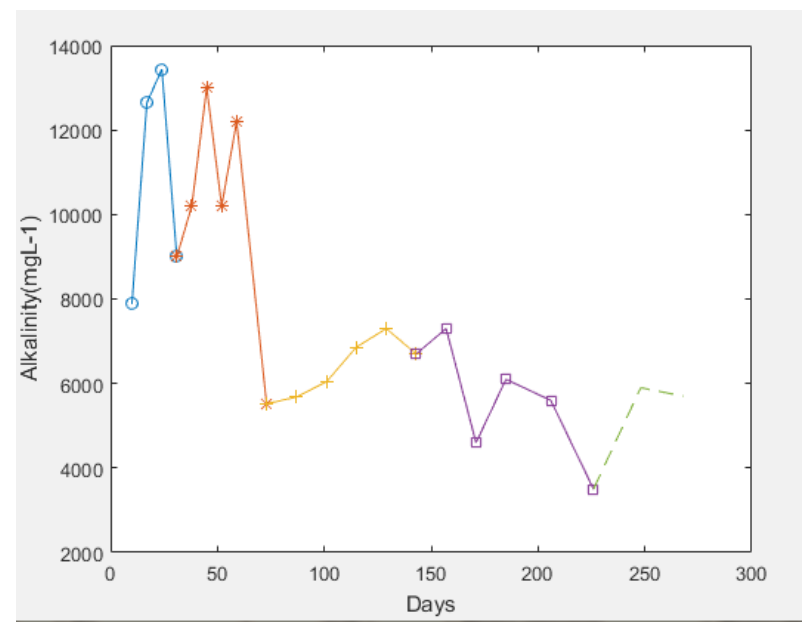

Figure 15:- Alkalinity transitory phase distribution
Coefficients in parameter Equations for R4 reactor by using transitory method:

Table 2: Equation for $\mathrm{pH}$

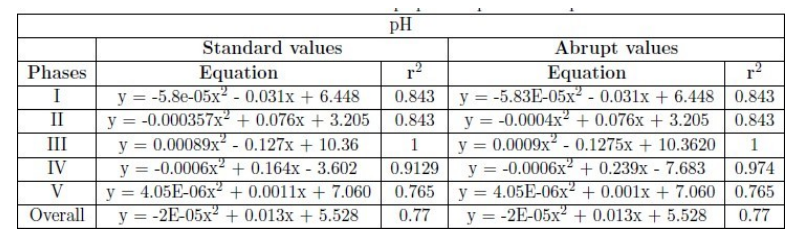

Table 3: Equation for EC

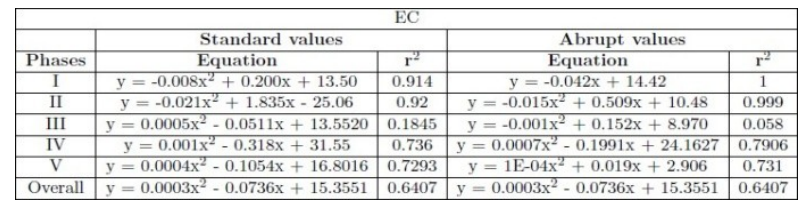

Table 4: Equation for TDS

\begin{tabular}{|c|c|c|c|c|}
\hline \multicolumn{5}{|c|}{ TDS } \\
\hline & Standard values & \multicolumn{2}{c|}{ Abrupt values } \\
\hline Phases & Equation & $\mathrm{r}^{2}$ & Equation & $\mathrm{r}^{2}$ \\
\hline I & $\mathrm{y}=-12.30 \mathrm{x}^{2}+349.7 \mathrm{x}+23292$ & 0.651 & $\mathrm{y}=-8.775 \mathrm{x}^{2}+371.2 \mathrm{x}+22925$ & 1 \\
\hline II & $\mathrm{y}=11.93 \mathrm{x}^{2}-1380 . \mathrm{x}+54607$ & 0.934 & $\mathrm{y}=-0.502 \mathrm{x}^{2}-295.7 \mathrm{x}+31574$ & 0.935 \\
\hline III & $\mathrm{y}=-10.75 \mathrm{x}^{2}+1572 . \mathrm{x}-38011$ & 0.752 & $\mathrm{y}=-10.75 \mathrm{x}^{2}+1572 . \mathrm{x}-38011$ & 0.752 \\
\hline IV & $\mathrm{y}=3.961 \mathrm{x}^{2}-1108 . \mathrm{x}+83839$ & 0.943 & $\mathrm{y}=3.961 \mathrm{x}^{2}-1108 . \mathrm{x}+83839$ & 0.943 \\
\hline V & $\mathrm{y}=0.039 \mathrm{x}^{2}-26.65 \mathrm{x}+10384$ & 0.88 & $\mathrm{y}=0.039 \mathrm{x}^{2}-26.65 \mathrm{x}+10384$ & 0.88 \\
\hline Overall & $\mathrm{y}=0.440 \mathrm{x}^{2}-194.2 \mathrm{x}+27094$ & 0.913 & $\mathrm{y}=0.440 \mathrm{x}^{2}-194.2 \mathrm{x}+27094$ & 0.913 \\
\hline
\end{tabular}

Table 5: Equation for BOD

\begin{tabular}{|c|c|c|c|c|}
\multicolumn{5}{|c|}{ BOD } \\
\hline & Standard values & Abrupt values \\
\hline Phase & Equation & $\mathrm{r}^{2}$ & Equation & $\mathrm{r}^{2}$ \\
\hline I & $\mathrm{y}=-11.13 \mathrm{x}^{2}+939.0 \mathrm{x}+19795$ & 0.371 & $\mathrm{y}=3314 . \mathrm{x}+7656$ & 1 \\
\hline II & $\mathrm{y}=23.07 \mathrm{x}^{2}-3187 . \mathrm{x}+11751$ & 0.979 & $\mathrm{y}=114.1 \mathrm{x}^{2}-4549 . \mathrm{x}+73202$ & 0.697 \\
\hline III & $\mathrm{y}=-0.388 \mathrm{x}^{2}-215.7 \mathrm{x}+28512$ & 0.802 & $\mathrm{y}=23.07 \mathrm{x}^{2}-3187 . \mathrm{x}+11751$ & 0.979 \\
\hline IV & $\mathrm{y}=1.541 \mathrm{x}^{2}-516.9 \mathrm{x}+42755$ & 0.709 & $\mathrm{y}=1.12 \mathrm{x}^{2}-389.3 \mathrm{x}+33590$ & 0.888 \\
\hline V & $\mathrm{y}=0.022 \mathrm{x}^{2}-10.17 \mathrm{x}+1235$ & 0.593 & $\mathrm{y}=0.024 \mathrm{x}^{2}-11.38 \mathrm{x}+1410$ & 0.775 \\
\hline Overall & $\mathrm{y}=0.900 \mathrm{x}^{2}-368.9 \mathrm{x}+35951$ & 0.796 & $\mathrm{y}=0.900 \mathrm{x}^{2}-368.9 \mathrm{x}+35951$ & 0.796 \\
\hline
\end{tabular}

Table 6: Equation of COD

\begin{tabular}{|c|c|c|c|c|}
\hline \multicolumn{5}{|c|}{ COD } \\
\hline & Standard values & Abrupt values \\
\hline Phases & Equation & $\mathrm{r}^{2}$ & Equation & $\mathrm{r}^{2}$ \\
\hline I & $\mathrm{y}=87.64 \mathrm{x}^{2}-2113 . \mathrm{x}+42393$ & 0.695 & $\mathrm{y}=1675 \mathrm{x}+264$ & 1 \\
\hline II & $\mathrm{y}=89.74 \mathrm{x}^{2}-8733 . \mathrm{x}+24982$ & 0.952 & $\mathrm{y}=322.4 \mathrm{x}^{2}-11313 \mathrm{x}+124$ & 1 \\
\hline III & $\mathrm{y}=15.79 \mathrm{x}^{2}-2925 . \mathrm{x}+15026$ & 0.969 & $\mathrm{y}=-66.73 \mathrm{x}^{2}+4691 . \mathrm{x}-297$ & 0.384 \\
\hline IV & $\mathrm{y}=3.598 \mathrm{x}^{2}-1134 . \mathrm{x}+900$ & 0.834 & $\mathrm{y}=3.257 \mathrm{x}^{2}-1041 . \mathrm{x}+83513$ & 0.955 \\
\hline V & $\mathrm{y}=0.022 \mathrm{x}^{2}-15.55 \mathrm{x}+3217$ & 0.874 & $\mathrm{y}=0.114 \mathrm{x}^{2}-58.70 \mathrm{x}+8211$ & 0.988 \\
\hline Overall & $\mathrm{y}=0.915 \mathrm{x}^{2}-433.5 \mathrm{x}+50004$ & 0.761 & $\mathrm{y}=0.915 \mathrm{x}^{2}-433.5 \mathrm{x}+500$ & 0.761 \\
\hline
\end{tabular}

Table 7: Equation for VFA

\begin{tabular}{|c|c|c|c|c|}
\hline \multicolumn{5}{|c|}{ VFA } \\
\hline & Standard values & \multicolumn{2}{c|}{ Abrupt values } \\
\hline Phases & Equation & $\mathrm{r}^{2}$ & Equation & $\mathrm{r}^{2}$ \\
\hline I & $\mathrm{y}=6.246 \mathrm{x}^{2}+68.04 \mathrm{x}+12155$ & 0.995 & $\mathrm{y}=2.840 \mathrm{x}^{2}+150.4 \mathrm{x}+11838$ & 0.998 \\
\hline II & $\mathrm{y}=63.19 \mathrm{x}^{2}-5970 \mathrm{x}+14628$ & 0.78 & $\mathrm{y}=-89.86 \mathrm{x}^{2}+5438 . \mathrm{x}-61739$ & 0.999 \\
\hline III & $\mathrm{y}=-45.94 \mathrm{x}^{2}+6511 . \mathrm{x}-20704$ & 0.967 & $\mathrm{y}=-34.83 \mathrm{x}^{2}+4911 . \mathrm{x}-15119$ & 0.939 \\
\hline IV & $\mathrm{y}=4.382 \mathrm{x}^{2}-1231 . \mathrm{x}+85771$ & 0.942 & $\mathrm{y}=5.397 \mathrm{x}^{2}-1456 . \mathrm{x}+97898$ & 0.943 \\
\hline V & $\mathrm{y}=0.011 \mathrm{x}^{2}-4.946 \mathrm{x}+552.9$ & 0.284 & $\mathrm{y}=0.022 \mathrm{x}^{2}-9.907 \mathrm{x}+1089$ & 0.674 \\
\hline Overall & $\mathrm{y}=0.250 \mathrm{x}^{2}-138.7 \mathrm{x}+17930$ & 0.597 & $\mathrm{y}=0.250 \mathrm{x}^{2}-138.7 \mathrm{x}+17930$ & 0.597 \\
\hline
\end{tabular}

Table 8: Equation for Alkalinity

\begin{tabular}{|c|c|c|c|c|}
\hline \multicolumn{5}{|c|}{ Alkalinity } \\
\hline & Standard values & Abrupt values \\
\hline Phases & Equation & $\mathrm{r}^{2}$ & Equation & $\mathrm{r}^{2}$ \\
\hline I & $\mathrm{y}=-42.15 \mathrm{x}^{2}+1444 . \mathrm{x}+3099$. & 0.57 & $\mathrm{y}=109.8 \mathrm{x}^{2}-1375 . \mathrm{x}+11660$ & 1 \\
\hline II & $\mathrm{y}=8.600 \mathrm{x}^{2}-366.5 \mathrm{x}+11149$ & 0.78 & $\mathrm{y}=29.88 \mathrm{x}^{2}-2148 . \mathrm{x}+47103$ & 0.857 \\
\hline III & $\mathrm{y}=9.191 \mathrm{x}^{2}-1517 . \mathrm{x}+69967$ & 0.998 & $\mathrm{y}=7.452 \mathrm{x}^{2}-1282 . \mathrm{x}+62261$ & 0.993 \\
\hline IV & $\mathrm{y}=0.603 \mathrm{x}^{2}-192.6 \mathrm{x}+20286$ & 0.628 & $\mathrm{y}=1.050 \mathrm{x}^{2}-329.9 \mathrm{x}+30445$ & 0.728 \\
\hline V & $\mathrm{y}=-0.045 \mathrm{x}^{2}+15.41 \mathrm{x}+3606$ & 0.403 & $\mathrm{y}=0.069 \mathrm{x}^{2}-39.26 \mathrm{x}+10015$ & 0.754 \\
\hline Overall & $\mathrm{y}=0.130 \mathrm{x}^{2}-69.06 \mathrm{x}+13300$ & 0.54 & $\mathrm{y}=0.130 \mathrm{x}^{2}-69.06 \mathrm{x}+13300$ & 0.54 \\
\hline
\end{tabular}


Coefficients in parameter Equations for R5 reactor by using transitory method:

Table 9: Equation for $\mathrm{pH}$

\begin{tabular}{|c|c|c|c|c|}
\hline \multicolumn{5}{|c|}{$\mathrm{pH}$} \\
\hline & Standard values & \multicolumn{2}{c|}{ Abrupt values } \\
\hline Phases & Equation & $\mathrm{r}^{2}$ & Equation & $\mathrm{r}^{2}$ \\
\hline I & $\mathrm{y}=-6 \mathrm{E}-05 \mathrm{x}^{2}-0.031 \mathrm{x}+6.448$ & 0.843 & $\mathrm{y}=-6 \mathrm{E}-05 \mathrm{x}^{2}-0.031 \mathrm{x}+6.448$ & 0.843 \\
\hline II & $\mathrm{y}=-0.0004 \mathrm{x}^{2}+0.076 \mathrm{x}+3.205$ & 0.843 & $\mathrm{y}=-0.0004 \mathrm{x}^{2}+0.076 \mathrm{x}+3.205$ & 0.843 \\
\hline III & $\mathrm{y}=0.0009 \mathrm{x}^{2}-0.127 \mathrm{x}+10.36$ & 1 & $\mathrm{y}=0.0009 \mathrm{x}^{2}-0.127 \mathrm{x}+10.36$ & 1 \\
\hline IV & $\mathrm{y}=-0.0006 \mathrm{x}^{2}+0.164 \mathrm{x}-3.602$ & 0.9129 & $\mathrm{y}=-0.0009 \mathrm{x}^{2}+0.239 \mathrm{x}-7.683$ & 0.974 \\
\hline V & $\mathrm{y}=4 \mathrm{E}-06 \mathrm{x}^{2}+0.001 \mathrm{x}+7.060$ & 0.765 & $\mathrm{y}=4 \mathrm{E}-06 \mathrm{x}^{2}+0.001 \mathrm{x}+7.060$ & 0.765 \\
\hline Overall & $\mathrm{y}=-2 \mathrm{E}-05 \mathrm{x}^{2}+0.013 \mathrm{x}+5.528$ & 0.77 & $\mathrm{y}=-2 \mathrm{E}-05 \mathrm{x}^{2}+0.013 \mathrm{x}+5.528$ & 0.77 \\
\hline
\end{tabular}

Table 10: Equation for EC

\begin{tabular}{|c|c|c|c|c|}
\hline \multicolumn{5}{|c|}{ SC } \\
\hline & Standard values & \multicolumn{2}{c|}{ Abrupt values } \\
\hline Phases & Equation & $\mathrm{r}^{2}$ & Equation & $\mathrm{r}^{2}$ \\
\hline I & $\mathrm{y}=-0.027 \mathrm{x}^{2}+1.035 \mathrm{x}+7.810$ & 0.938 & $\mathrm{y}=-0.017 \mathrm{x}^{2}+1.447 \mathrm{x}-13.84$ & 0.836 \\
\hline II & $\mathrm{y}=-0.027 \mathrm{x}^{2}+2.248 \mathrm{x}-29.36$ & 0.843 & $\mathrm{y}=-0.027 \mathrm{x}^{2}+1.035 \mathrm{x}+7.810$ & 0.938 \\
\hline III & $\mathrm{y}=-0.0004 \mathrm{x}^{2}+0.0128 \mathrm{x}+14.3318$ & 0.264 & $\mathrm{y}=-0.0005 \mathrm{x}^{2}+0.0844 \mathrm{x}+9.8089$ & 0.3658 \\
\hline IV & $\mathrm{y}=5 \mathrm{E}-05 \mathrm{x}^{2}-0.018 \mathrm{x}+13.54$ & 0.02 & $\mathrm{y}=-0.001 \mathrm{x}^{2}+0.573 \mathrm{x}-39.54$ & 0.563 \\
\hline V & $\mathrm{y}=0.000 \mathrm{x}^{2}-0.154 \mathrm{x}+23.42$ & 0.812 & $\mathrm{y}=-0.002 \mathrm{x}^{2}+1.339 \mathrm{x}-152.4$ & 0.92 \\
\hline Overall & $\mathrm{y}=0.0004 \mathrm{x}^{2}-0.1046 \mathrm{x}+18.7111$ & 0.7018 & $\mathrm{y}=0.0004 \mathrm{x}^{2}-0.1046 \mathrm{x}+18.7111$ & 0.7018 \\
\hline
\end{tabular}

Table 11: Equation for TDS

\begin{tabular}{|c|c|c|c|c|}
\hline \multicolumn{5}{|c|}{ TDS } \\
\hline & Standard values & \multicolumn{2}{c|}{ Abrupt values } \\
\hline Phase & Equation & $\mathrm{r}^{2}$ & Equation & $\mathrm{r}^{2}$ \\
\hline I & $\mathrm{y}=-14.59 \mathrm{x}^{2}+229.9 \mathrm{x}+23736$ & 0.481 & $\mathrm{y}=622.8 \mathrm{x}+16971$ & 1 \\
\hline II & $\mathrm{y}=-2.959 \mathrm{x}^{2}-11.81 \mathrm{x}+21253$ & 0.963 & $\mathrm{y}=43.98 \mathrm{x}^{2}-2892 . \mathrm{x}+63226$ & 0.85 \\
\hline III & $\mathrm{y}=0.238 \mathrm{x}^{2}-126.1 \mathrm{x}+17963$ & 0.855 & $\mathrm{y}=5.376 \mathrm{x}^{2}-773.8 \mathrm{x}+38182$ & 0.931 \\
\hline IV & $\mathrm{y}=0.661 \mathrm{x}^{2}-160 \mathrm{x}+17397$ & 0.754 & $\mathrm{y}=-0.014 \mathrm{x}^{2}-22.77 \mathrm{x}+11200$ & 0.536 \\
\hline V & $\mathrm{y}=0.338 \mathrm{x}^{2}-146.7 \mathrm{x}+22618$ & 0.197 & $\mathrm{y}=-0.678 \mathrm{x}^{2}+326 \mathrm{x}-31562$ & 0.703 \\
\hline Overall & $\mathrm{y}=0.542 \mathrm{x}^{2}-198.4 \mathrm{x}+23681$ & 0.845 & $\mathrm{y}=0.542 \mathrm{x}^{2}-198.4 \mathrm{x}+23681$ & 0.845 \\
\hline
\end{tabular}

Table 12: Equation for BOD

\begin{tabular}{|c|c|c|c|c|}
\hline \multicolumn{5}{|c|}{ BOD } \\
\hline & Standard values & \multicolumn{2}{c|}{ Abrupt values } \\
\hline Phases & Equation & $\mathrm{r}^{2}$ & Equation & $\mathrm{r}^{2}$ \\
\hline I & $\mathrm{y}=131.4 \mathrm{x}^{2}-4450 \mathrm{x}+54344$ & 0.997 & $\mathrm{y}=-1027 . \mathrm{x}+33480$ & 1 \\
\hline II & $\mathrm{y}=15.18 \mathrm{x}^{2}-2224 . \mathrm{x}+96373$ & 0.984 & $\mathrm{y}=-60.19 \mathrm{x}^{2}+4276 . \mathrm{x}-39860$ & 0.782 \\
\hline III & $\mathrm{y}=-1.982 \mathrm{x}^{2}-398.1 \mathrm{x}+50298$ & 0.864 & $\mathrm{y}=5.729 \mathrm{x}^{2}-1364 . \mathrm{x}+79561$ & 0.901 \\
\hline IV & $\mathrm{y}=0.567 \mathrm{x}^{2}-160.1 \mathrm{x}+11348$ & 0.988 & $\mathrm{y}=-0.245 \mathrm{x}^{2}+75.77 \mathrm{x}-5704$ & 1 \\
\hline V & $\mathrm{y}=0.004 \mathrm{x}^{2}-2.299 \mathrm{x}+386.6$ & 0.509 & $\mathrm{y}=-0.003 \mathrm{x}^{2}+1.141 \mathrm{x}+2.420$ & 0.287 \\
\hline Overall & $\mathrm{y}=0.944 \mathrm{x}^{2}-378.6 \mathrm{x}+35682$ & 0.734 & $\mathrm{y}=0.944 \mathrm{x}^{2}-378.6 \mathrm{x}+35682$ & 0.734 \\
\hline
\end{tabular}

Table 13: Equation for COD

\begin{tabular}{|c|c|c|c|c|}
\hline \multicolumn{5}{|c|}{ COD } \\
\hline & Standard values & Abrupt values \\
\hline Phases & Equation & $\mathrm{r}^{2}$ & Equation & $\mathrm{r}^{2}$ \\
\hline I & $\mathrm{y}=172.3 \mathrm{x}^{2}-6604 . \mathrm{x}+83344$ & 0.927 & $\mathrm{y}=-2608 . \mathrm{x}+61766$ & 1 \\
\hline II & $\mathrm{y}=10.93 \mathrm{x}^{2}-540.7 \mathrm{x}+47532$ & 0.338 & $\mathrm{y}=-81.40 \mathrm{x}^{2}+5550 . \mathrm{x}-54740$ & 0.906 \\
\hline III & $\mathrm{y}=34.66 \mathrm{x}^{2}-6105 . \mathrm{x}+27321$ & 0.974 & $\mathrm{y}=-67.89 \mathrm{x}^{2}+6649 . \mathrm{x}-11509$ & 0.97 \\
\hline IV & $\mathrm{y}=0.842 \mathrm{x}^{2}-270.2 \mathrm{x}+22724$ & 0.958 & $\mathrm{y}=1.132 \mathrm{x}^{2}-354.9 \mathrm{x}+28624$ & 0.967 \\
\hline V & $\mathrm{y}=0.040 \mathrm{x}^{2}-20.27 \mathrm{x}+3237$ & 0.431 & $\mathrm{y}=-0.014 \mathrm{x}^{2}+4.463 \mathrm{x}+473.8$ & 0.155 \\
\hline Overall & $\mathrm{y}=1.053 \mathrm{x}^{2}-451.3 \mathrm{x}+46808$ & 0.679 & $\mathrm{y}=1.053 \mathrm{x}^{2}-451.3 \mathrm{x}+46808$ & 0.679 \\
\hline
\end{tabular}

Table 14: Equation for VFA

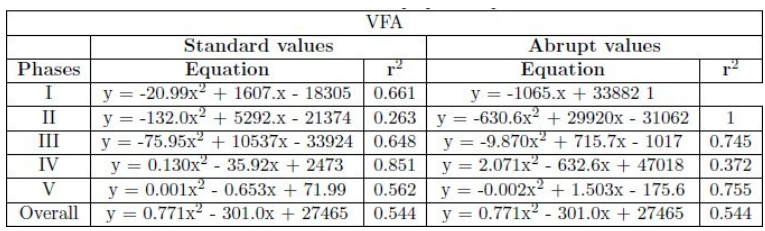

Table 15: Equation for Alkalinity

\begin{tabular}{|c|c|c|c|c|}
\hline \multicolumn{5}{|c|}{ Alkalinity } \\
\hline & Standard values & Abrupt values \\
\hline Phases & Equation & $\mathrm{r}^{2}$ & Equation & $\mathrm{r}^{2}$ \\
\hline I & $\mathrm{y}=-46.93 \mathrm{x}^{2}+1983 \mathrm{x}-7329$ & 0.996 & $\mathrm{y}=-46.93 \mathrm{x}^{2}+1983 . \mathrm{x}-7329$ & 0.996 \\
\hline II & $\mathrm{y}=-20.40 \mathrm{x}^{2}+1785 \mathrm{x}-27092$ & 0.7 & $\mathrm{y}=-10.64 \mathrm{x}^{2}+1035 \mathrm{x}-13141$ & 0.817 \\
\hline III & $\mathrm{y}=2.045 \mathrm{x}^{2}-463.4 \mathrm{x}+30039$ & 0.698 & $\mathrm{y}=-0.317 \mathrm{x}^{2}+92.11 \mathrm{x}+280.7$ & 0.817 \\
\hline IV & $\mathrm{y}=-0.408 \mathrm{x}^{2}+121.0 \mathrm{x}-1814$ & 0.825 & $\mathrm{y}=-0.250 \mathrm{x}^{2}+57.98 \mathrm{x}+3551$ & 0.602 \\
\hline V & $\mathrm{y}=0.512 \mathrm{x}^{2}-225.4 \mathrm{x}+29526$ & 0.364 & $\mathrm{y}=-2.835 \mathrm{x}^{2}+1453 \mathrm{x}-18007$ & 1 \\
\hline Overall & $\mathrm{y}=0.139 \mathrm{x}^{2}-63.26 \mathrm{x}+12411$ & 0.603 & $\mathrm{y}=0.139 \mathrm{x}^{2}-63.26 \mathrm{x}+12411$ & 0.603 \\
\hline
\end{tabular}

\subsection{Discussion}

It was observed major seven parameters from leachate chemical analysis which is $\mathrm{pH}, \mathrm{EC}, \mathrm{VFA}, \mathrm{BOD}, \mathrm{COD}$, TDS and alkalinity.

For all these seven parameters we have plotted graphs for standard and transitory phase distribution graphs. These graphs show the value of R2 which shows the perfect curve fit.

In standard and transitory phase distribution graph, nature of curve remains same but R2 Values are different because of different phase distribution methods. From the observation transitory method of phase distribution is best method. Hence by considering that method equations of each phase is obtained for reactor R4 and R5.

\section{Conclusion}

1. Obtained phase distribution for chemical parameters further phase equations are obtained.

2. Phase equations give phase values of parameters which defines quality and quantity of chemical parameter.

\section{References}

1. P. Bajpai, Basics of anaerobic digestion process, Anaerobic Technology, Springer, (2017).

2. S. T. Mali, K. C. Khare, A.H. Biradar, Enhancement of methane production and bio stabilization of municipal solid waste in anaerobic bioreactor landfill, Bio- resource Technology, Elsevier, vol. 110, pp. 10, 17, April (2012).

3. Vesilind PA, Worrell WA, Reinhart DR. Solid Waste engineering, Singapore: Thomoson Asia Pvt. Ltd, (2003). 